\title{
Spatial-Temporal Variability of Inertial Currents in the Eastern Part of the Black Sea in a Storm Period
}

\author{
N. A. Diansky ${ }^{1,2,3} *$, V. V. Fomin ${ }^{2}$, A. V. Grigoriev ${ }^{2,4}$, \\ A. V. Chaplygin ${ }^{1}$ A. G. Zatsepin ${ }^{4,5}$ \\ ${ }^{1}$ Lomonosov Moscow State University, Moscow, Russian Federation \\ ${ }^{2}$ Zubov State Oceanographic Institute, Moscow, Russian Federation \\ ${ }^{3}$ Marchuk Institute of Numerical Mathematics, Russian Academy of Sciences, \\ Moscow, Russian Federation \\ ${ }^{4}$ Moscow Institute of Physics and Technology (national research university), \\ Dolgoprudny, Russian Federation \\ ${ }^{5}$ Shirshov Institute of Oceanology, Russian Academy of Sciences, Moscow, Russian Federation \\ *nikolay.diansky@gmail.com
}

\begin{abstract}
Introduction. Analysis of numerical simulation of the inertial oscillations evolution in a storm period in the northeastern Black Sea is represented in comparison with the observations and the analytical solutions. The simulations confirm significant contribution of the inertial motions to formation of the velocity fields. It is shown that inertial oscillations do not affect the sea level changes and their character depends on vertical structure of the sea upper mixed layer.

Data and methods. Comparison the simulation results to the drift observations in the Black Sea were represented. It is noted that intensity of inertial oscillations in the deep-sea areas significantly exceeds the one in the shallow areas. At that, contribution of the inertial currents to the Black Sea general circulation in the deep-sea zone is comparable to that of the mesoscale motions. Analytical solution of the inertial oscillations' equation system (taking into account wind and its absence) was studied.

Results. Inertial oscillations are excited in the velocity module due to an abrupt change of the wind speed. Moreover, harmonic oscillations are disturbed with depth. After the wind affect is over, the current velocity is barotropized due to fast transition from a quasi-stationary state to another one. It is shown that inertial oscillations are of two time scales conditioned by vertical viscosity.

Discussion and conclusion. The first, short time scale is responsible for rapid formation of a new quasi-stationary state. It does not depend on the viscosity coefficient and is approximately equal to two inertial periods. The other, longer scale of the inertial oscillations attenuation, is associated with the energy drain of quasi-stationary oscillations from the upper layers of the sea to the deeper ones. This slow process of attenuation is proportional to the root of time.
\end{abstract}

Keywords: the Black Sea, inertial oscillations, Ekman theory, numerical simulation, upper mixed layer, analytical solution.

Acknowledgments: the authors are thankful to Head Scientist Researcher of State Oceanographic Institute E. V. Borisov for his important remarks on the paper. The investigation is carried out at support of Russian Scientific Foundation (grant No. 17-77-30001) and RFBR-RGS (grant No. 17-0541089).

For citation: Diansky, N.A., Fomin, V.V., Grigoriev, A.V., Chaplygin, A.V. and Zatsepin, A.G., 2019. Spatial-Temporal Variability of Inertial Currents in the Northeastern Part of the Black Sea. Physical Oceanography, [e-journal] 26(2), pp. 135-146. doi:10.22449/1573-160X-2019-2-135-146

DOI:10.22449/1573-160X-2019-2-135-146

(C) 2019, N. A. Diansky, V. V. Fomin, A. V. Grigoriev, A. V. Chaplygin, A. G. Zatsepin

(C) 2019, Physical Oceanography

\section{Introduction}

Storms in the Black Sea are quite common events [1-3], they are strongest in winter and also in transitional seasons - in spring and autumn. Such events include the strongest storm on March 23-24, 2013, when almost the entire sea area was on PHYSICAL OCEANOGRAPHY VOL. 26 ISS. 2 (2019) 
the periphery of a deep cyclone [1, 4]. From the analysis of drifter data, it follows $[5,6]$ that inertial oscillations make a significant contribution to the formation of a field of currents in the Black Sea and in periods of storms are not inferior in energy to mesoscale eddy motions. However, their intensity is different in various parts of the sea [6].

This paper presents the results of the numerical modeling data analysis of the Black Sea circulation during the storm, their physical interpretation, as well as the study of the analytical solution of the system of equations describing them.

\section{Description of the technology for obtaining the calculated felds of currents and their analysis}

The State Oceanographic Institute implemented the Operational Current Calculation System (OCCS) to solve the problem of calculating circulation in the waters of the Black and Azov Seas. For its operation, the Institute of Numerical Mathematics Ocean Model (INMOM) of the Russian Academy of Sciences [7] and the Weather Research Forecast $(W R F)$ regional non-hydrostatic model of atmospheric circulation [8] are used. Their characteristics and verification results are described in detail in $[1,9]$.

The study of the storm situation according to the results of calculations using the WRF model showed that wind speeds during the storm period exceeded $20 \mathrm{~m} / \mathrm{s}$ (Fig. 1). Nevertheless, since the bottom topography in the region has a steep character, the wind surge at the coast did not exceed $20 \mathrm{~cm}$ (Fig. 2).
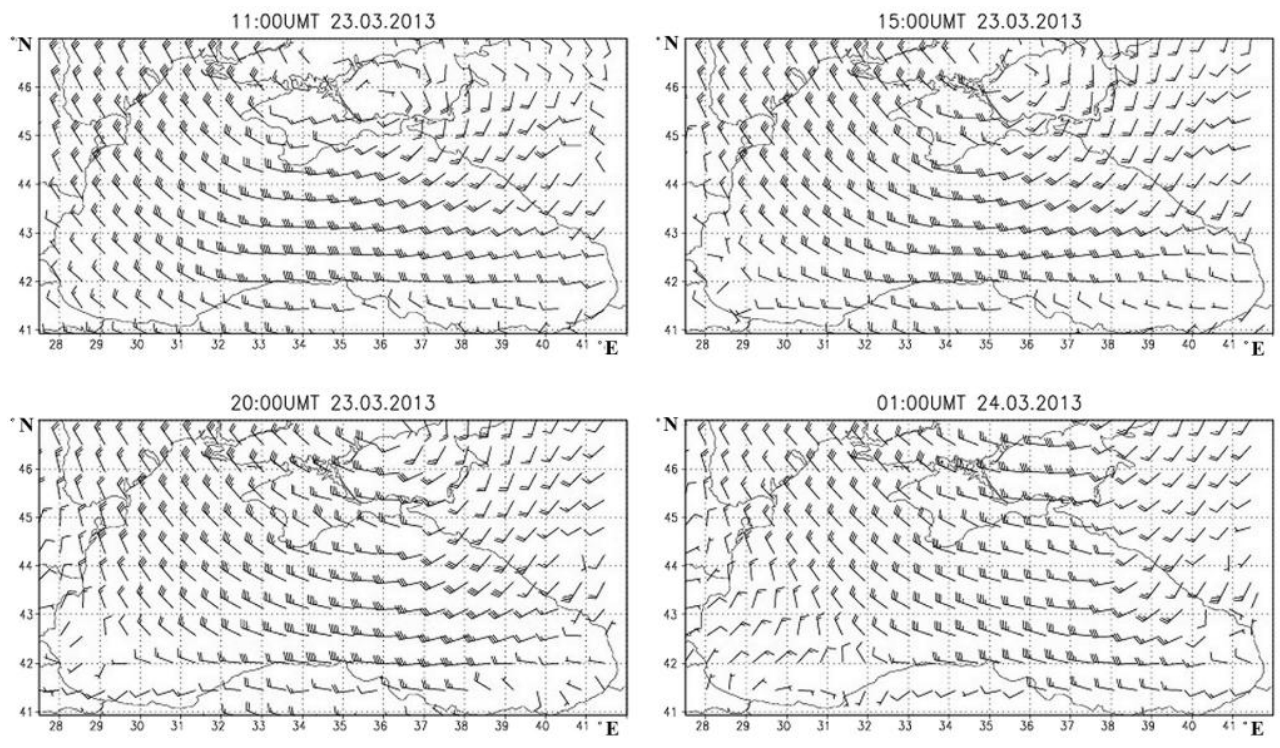

F i g. 1. Wind field over the Black Sea in different moments of storm evolution

As seen in Fig. 2, the currents show no correspondence with Ekman's stationary theory. In the open sea part, the velocity vectors turn clockwise with time and do not show a relation with the level gradients, which is typical of inertial flows [10-15]. Correspondence with the gradient level is visible only in coastal 
areas. Thus, in the sea area, inertial oscillations make the main contribution to the generation of currents in a storm situation.
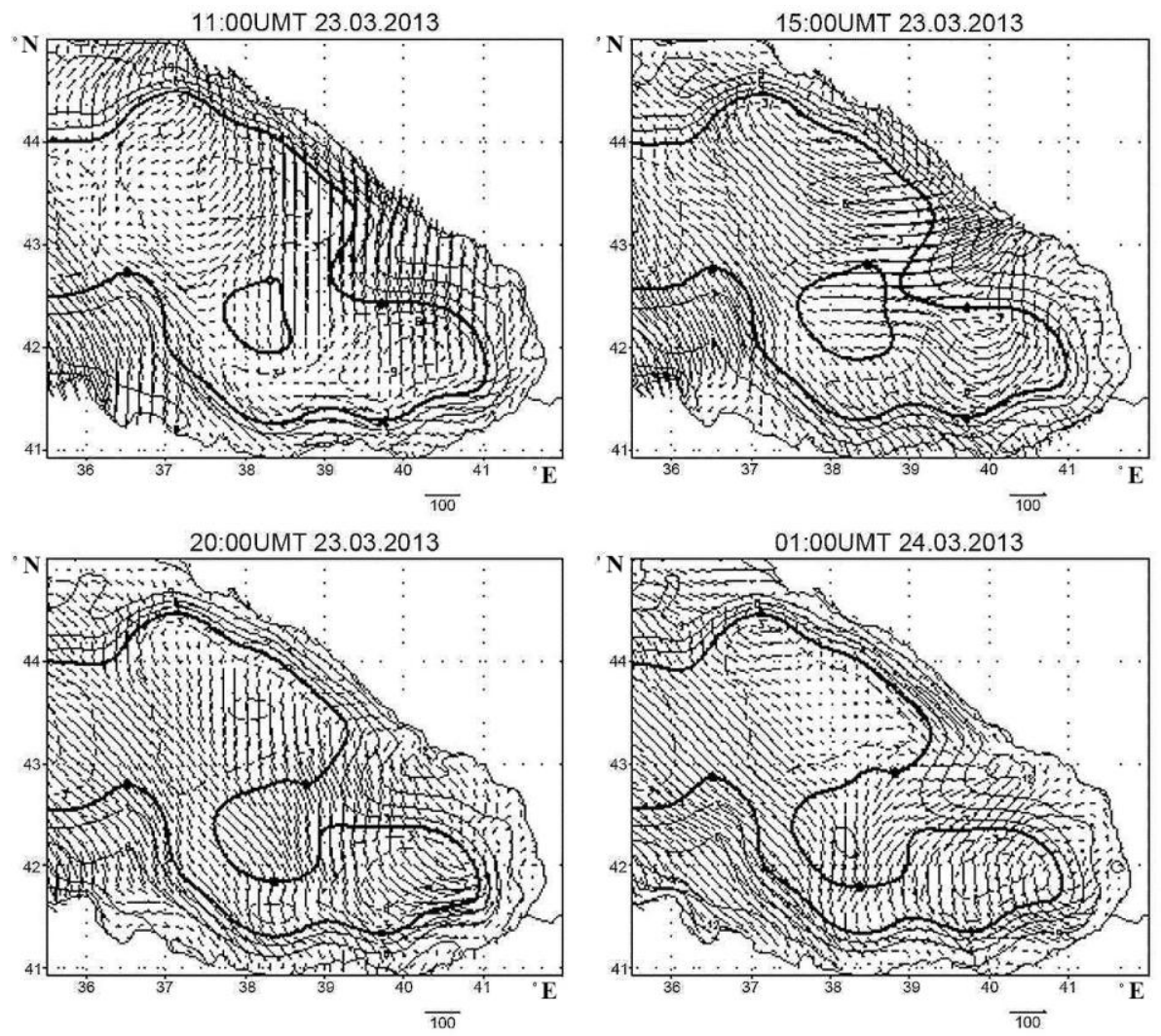

F i g. 2. Sea level isolines (solid lines - positive values and stroke ones - negative values) and currents velocities near the surface (arrows, $\mathrm{cm} / \mathrm{s}$ ) in the eastern Black Sea for the same time moments as on Fig. 1. Scale of the velocity vectors is under the figure

Analysis of the vertical structure of wind speed at a point with coordinates of $43.0^{\circ} \mathrm{N}, 39.2^{\circ} \mathrm{E}$ (Fig. 3), corresponding to the deep-sea area, shows that inertial oscillations with an appropriate latitude inertial period of $17.6 \mathrm{~h}$ are actually observed. At the same time, oscillations with inertial frequency are not reflected in wind speed [10], but there is a pronounced phase shift between the maxima of wind speed and speeds of inertial movements in the sea (Fig. 3), which confirms their inertial nature with respect to the driving force of the wind.

In [16] the amplitude of the velocity of inertial oscillations $U_{0}$ is estimated, which is average in the upper quasi-homogeneous layer (UQHL), depending on the wind speed:

$$
U_{0} \approx \frac{\tau_{0}}{\rho_{w} f H},
$$

where the wind friction stress is calculated using a bulk formula (see, for example, [7]); $\tau_{0}=\rho_{a} C_{D} W_{10}^{2}$ is the wind friction stress module with speed $W_{10}$ at the height of $10 \mathrm{~m} ; \rho_{w}$ and $\rho_{a}$ is water and air density, $C_{D}=\left(1,1+0,04 W_{10}\right) 10^{-3}$ is dynamic 
coefficient of friction; $f$ is the Coriolis parameter; $H$ is the OQHL thickness. Assuming that (according to Fig. 3) in (1) $W_{10} \approx 15 \mathrm{~m} / \mathrm{s}$, in the upper layer under $H=5 \mathrm{M}, U_{0} \approx 100 \mathrm{~cm} / \mathrm{s}$ is obtained, and in the layer $H=20 \mathrm{~m} U_{0} \approx 25 \mathrm{~cm} / \mathrm{s}$ is obtained, which agrees well with the calculations shown in Fig. 2 and 3.

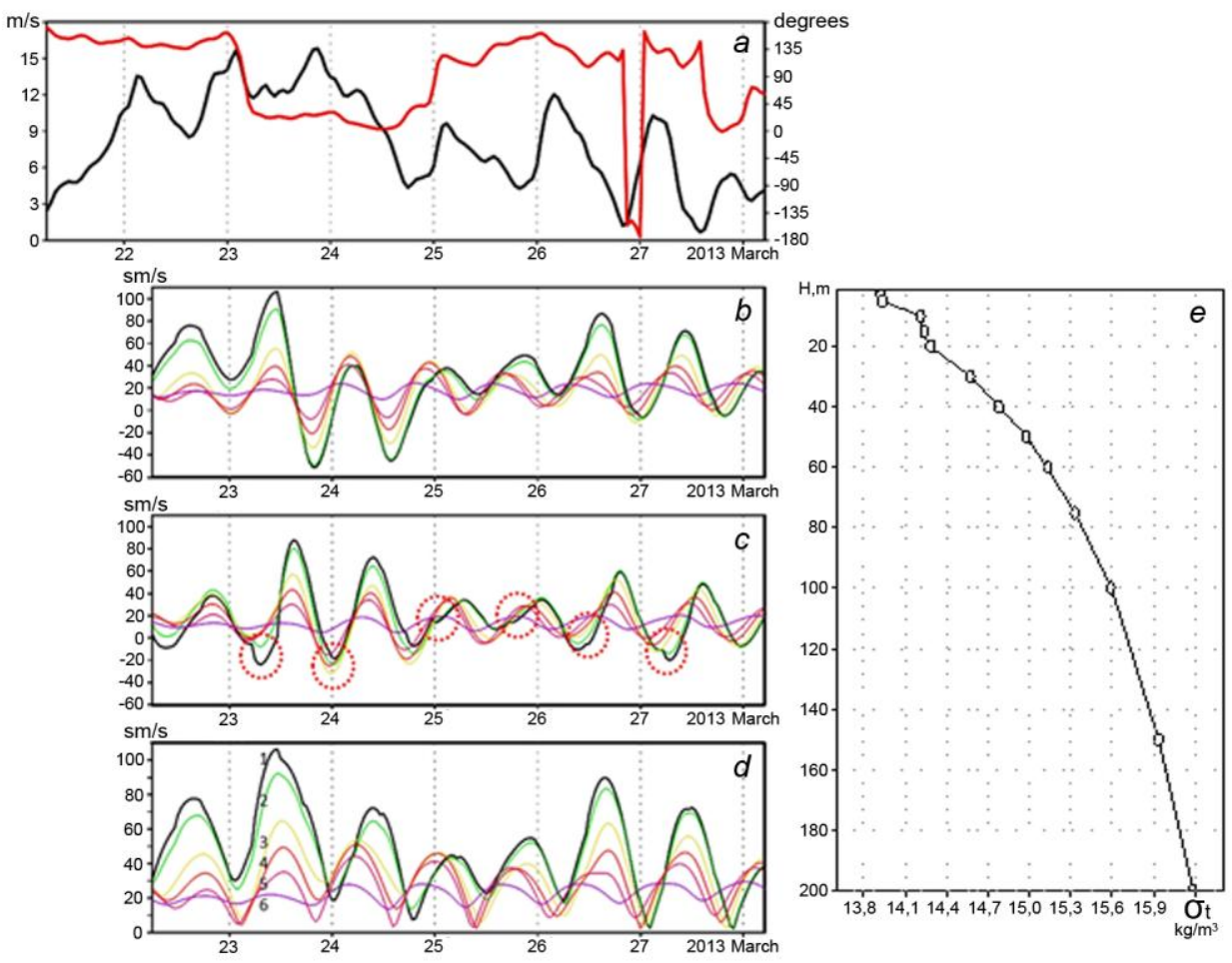

F i g. 3. Temporal variation of module ( $\mathrm{m} / \mathrm{s}$, black line) and direction (degrees, red line) of wind speed $(a)$, meridian $(b)$ and zonal $(c)$ components $(\mathrm{cm} / \mathrm{s})$ and module of currents velocity $(d)$ in the point $43.0^{\circ} \mathrm{N}, 39.2^{\circ} \mathrm{E}$ (velocities on the depths are shown by colors and numbers: $1 \mathrm{~m}$ - curve 1 ; $5 \mathrm{~m}$ - curve 2;10 m - curve 3;15 m-curve 4;20 m-curve 5;30 m - curve 6) and also vertical profile of conventional density $\sigma_{t}\left(\mathrm{~kg} / \mathrm{m}^{3}\right)$ in the very same point $(e)$

According to the vertical density profile at the point of analysis, two elevated gradients of quasi-homogeneous layers separated by a zone can be distinguished: the first one - at a depth of 0-7.5 m, the second - at a depth of 9-25 m (model data, Fig. 3e). It is seen that the values of the amplitudes of the inertial velocities are grouped within these layers [17]. With a slight change in wind exposure, inertial oscillations change only in the upper layer, remaining the same in the lower one. Such changes in the velocity in the upper part of the QHL disagreed with the changes in the lower part of the QHL are marked by circles in Fig. 3. Changes in wind speed correspond to these time points (Fig. 3a). 

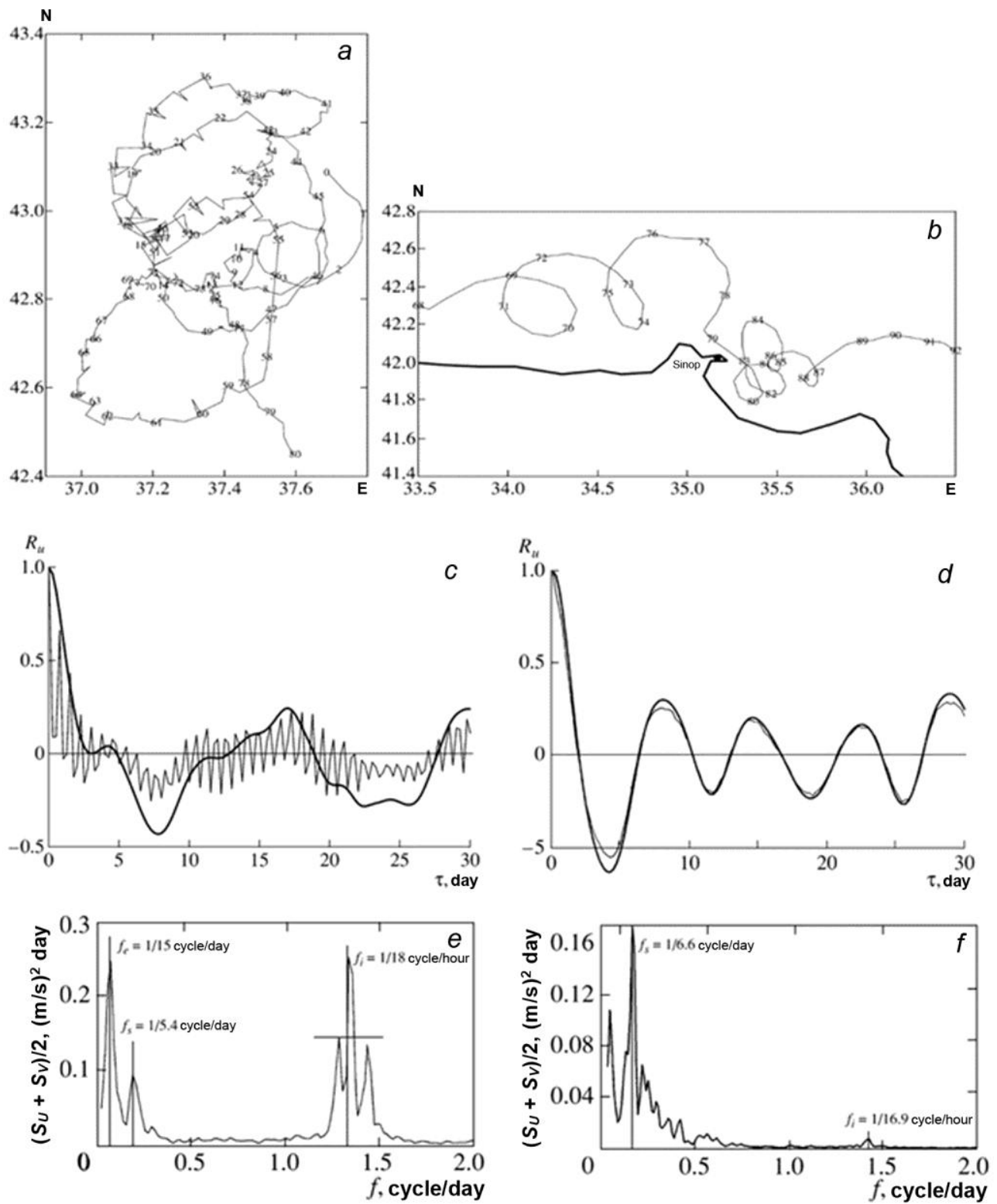

F i g. 4. Fragments of trajectories of drifters No. $17430(a)$ and No. 16331 (b) (numbers on the trajectories denote the number of days since a drifter launch); normalized autocorrelations $R$ of zonal component of drifters No. $17430(c)$ and No. 16331 velocities (d) taking into account (thin line) and taking no account (solid line) of inertial oscillations; total spectral density of velocity components $u$ and $v$ of drifters No. $17430(e)$ and No. 16331(f) [6]

The amplitude of inertial oscillations decreases with depth, and their harmonic character is distorted (Fig. $3 d$ ). The inertial oscillations practically do not penetrate below the density jump, to a depth of $30 \mathrm{~m}$, having an amplitude of less than $5 \mathrm{~cm} / \mathrm{s}$. 


\section{Comparison with field observations}

The peculiarities of the Black Sea circulation in the upper layer at different time scales [5] can be illustrated, in particular, by the results of the international drifter experiment 1999-2002 published in [6]. As already shown above, according to the results of a numerical experiment, inertial oscillations in the open part of the sea are pronounced. This is in good agreement with the data of drifter observations. Fig. 4 shows the results of these drifters, the trajectories of which are localized in the deep-water (drifter No. 17430) and relatively shallow-water (drifter No. 16331) areas of the sea. It can be seen that in the deep-sea area the inertial oscillations of the velocity of currents in the upper layer are pronounced much more clearly in comparison with the shallow-water region and are comparable in energy with mesoscale movements [6]. Presented in Fig. 3, the simulation results of currents at the analysis point refer to the deep-water zone. This suggests that the inertial currents here should be well manifested, which follows from the figure.

\section{Analytical study of the behavior of inertial oscillations}

Fig. $3 d$ shows the loss of the harmonic nature of the velocity module fluctuations with depth; while in the velocity components the harmonic nature is preserved at all depths. To find out the cause, the behavior of inertial oscillations based on an analytical solution of the system of equations describing them is considered.

Drift currents (Ekman and inertia ones) are described by a simple system of linear equations.

$$
\begin{aligned}
& \frac{\partial u}{\partial t}-f v=v \frac{\partial^{2} u}{\partial z^{2}}, \\
& \frac{\partial v}{\partial t}+f u=v \frac{\partial^{2} v}{\partial z^{2}},
\end{aligned}
$$

where $\boldsymbol{u}$ and $\boldsymbol{v}$ are zonal and meridional components of the velocity of currents, depending on the depth $z$ and time $t ; v$ is the kinematic coefficient of the vertical turbulent viscosity.

Properties of the solution to the system of equations (2) are convenient to consider when setting simplified boundary conditions. It is as if the western wind, starting from some point $t=0$, is constant in speed $\left(W_{10}=15 \mathrm{M} / \mathrm{c}\right)$ and direction so that the wind friction stress components are $\tau_{x}>0$ and $\tau_{y}=0$. Further, when the decision is established (in this case this period is conventionally assumed to be 15 days), the wind instantly turns to zero.

If the velocity of currents is imagined in a complex form $w(z, t)=u(z, t)+i v(z, t)$, then the system of equations (2) can be expressed by the only one equation as follows

$$
\frac{\partial w}{\partial t}+\text { if } w=v \frac{\partial^{2} w}{\partial z^{2}}
$$


with general boundary conditions

$$
\begin{gathered}
\left.v \frac{\partial w}{\partial z}\right|_{z=0}=-\tau_{x}(t)-i \tau_{y}(t), \\
\left.w\right|_{z \rightarrow \infty}=0 \\
w(z, 0)=w_{0}(z) .
\end{gathered}
$$

Substitution in (3) of $w$ by $w(z, t)=W(z, t) e^{-i f t}$, where $W(z, t)$ is the amplitude of inertial oscillations, results in the classical diffusion equation

$$
\frac{\partial W}{\partial t}=v \frac{\partial^{2} W}{\partial z^{2}}
$$

for the amplitude (or velocity module) of inertial motions with their own boundary conditions, which follow from (4). Equation (5) describes the amplitude decay with depth and in the stationary case - it follows a linear law.

Solving the given problem of excitation and attenuation of inertial oscillations, the boundary and initial conditions divide the general problem (3) - (5) into two subtasks. The first describes the occurrence of inertial oscillations under the western wind action with boundary conditions

$$
\begin{gathered}
\left.v \frac{\partial W}{\partial z}\right|_{z=0}=-\tau_{z} e^{i f t}, \\
\left.W\right|_{z \rightarrow \infty}=0, \\
W(z, 0)=0,
\end{gathered}
$$

the second - is the attenuation of inertial movements in the absence of wind:

$$
\begin{gathered}
\left.\mathrm{v} \frac{\partial W}{\partial z}\right|_{z=0}=0, \\
\left.W\right|_{z \rightarrow \infty}=0, \\
W\left(z, t_{15}\right)=M(z),
\end{gathered}
$$

where $M(z)$ is the solution of problem (5) and (6) on the $15^{\text {th }}$ day.

It should be noted that by virtue of (6) and (7) the amplitude of forced inertial oscillations (under the wind action) will be complex and free (in the absence of wind) - real, i.e. the amplitude of forced inertial oscillations will also experience (see below ) close to harmonic oscillations at inertial frequency.

The solution of the first problem (5) and (6) for the module of inertial movements is written in the form [13]

$$
W_{1}(z, t)=\frac{\tau_{x}}{\sqrt{v \pi}} \int_{0}^{t} \frac{1}{\sqrt{\zeta}} \exp \left(-\frac{z^{2}}{4 v \zeta^{2}}-i f \zeta\right) d \zeta
$$

or for components of speed - in the form obtained also in [13]:

$$
\begin{aligned}
& u_{1}(z, t)=\frac{\tau_{x}}{\sqrt{v \pi}} \int_{0}^{t} \frac{1}{\sqrt{\zeta}} \exp \left(-\frac{z^{2}}{4 v_{\zeta}}\right) \cos (f \zeta) d \zeta, \\
& v_{1}(z, t)=-\frac{\tau_{x}}{\sqrt{v \pi}} \int_{0}^{t} \frac{1}{\sqrt{\zeta}} \exp \left(-\frac{z^{2}}{4 v \zeta}\right) \sin (f \zeta) d \zeta .
\end{aligned}
$$


On the sea surface, the solution (9) is reduced to a simpler form:

$$
\begin{gathered}
u_{1}(0, t)=\frac{2 \tau_{x}}{\sqrt{v \pi f}} C(\sqrt{f t}) \approx \frac{2 \tau_{x}}{\sqrt{v \pi f}}\left(\sqrt{\frac{\pi}{2}}+\frac{1}{\sqrt{f t}} \sin (f t)\right), \\
v_{1}(0, t)=-\frac{2 \tau_{x}}{\sqrt{v \pi f}} S(\sqrt{f t}) \approx-\frac{2 \tau_{x}}{\sqrt{v \pi f}}\left(\sqrt{\frac{\pi}{2}}-\frac{1}{\sqrt{f t}} \cos (f t)\right),
\end{gathered}
$$

where $C(\psi)=\int_{0}^{\psi} \cos \left(t^{2}\right) d t$ and $S(\psi)=\int_{0}^{\psi} \sin \left(t^{2}\right) d t$ are Fresnel integrals, determining the behavior of inertial velocities on the sea surface with a phase shift between $u$ and $\pi / 2$, are clearly visible in Fig. 3 and 5 .

The second problem (6) and (7) is solved in the following way. When $t=t_{15}$ the (5) and (7) problem solution will be expressed as follows

$$
M(z)=\frac{\tau_{x}}{\sqrt{v \pi}} \int_{0}^{t_{15}} \frac{1}{\sqrt{\zeta}} \exp \left(-\frac{z^{2}}{4 v \zeta}-i f \zeta\right) d \zeta .
$$
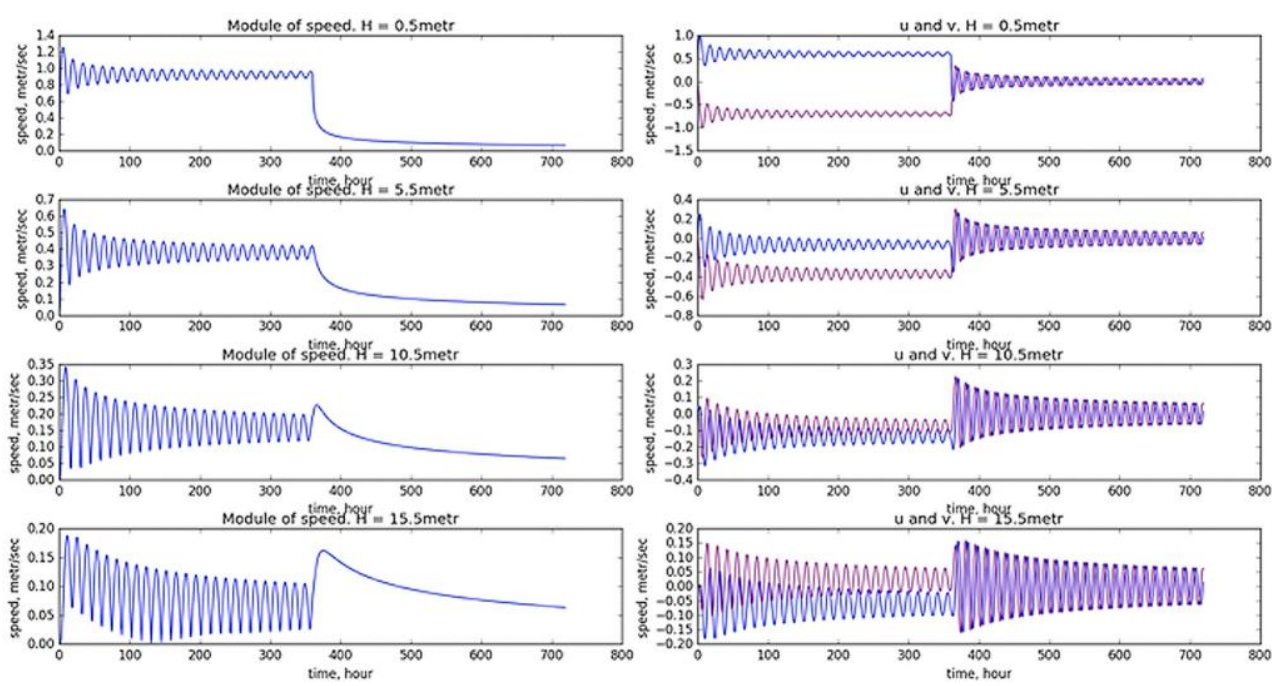

F i g. 5. Graphs of analytical solution of the inertial oscillations equation system with wind pumping and without it (blue curve - wind speed zonal component, purple one - wind speed meridian component)

Then the general solution of problem (5) and (7) for the amplitude of free inertial motions is written through the Poisson integral [18]:

$W_{2}(z, t)=\frac{1}{2 \sqrt{\pi v\left(t-t_{15}\right)}} \int_{0}^{+\infty}\left[\exp \left(-\frac{(z-s)^{2}}{4 v\left(t-t_{15}\right)}\right)+\exp \left(-\frac{(z+s)^{2}}{4 v\left(t-t_{15}\right)}\right)\right] M(s) d s,(12)$

where $t \geq t_{15}$. In this case, the solution of the problem due to the vertical viscosity will be rearranged from one solution to another. This time can be estimated as $t_{E} \approx d_{E}{ }^{2} / v=2 / f$, where $d_{E}=\sqrt{\frac{2 v}{f}}$ is the characteristic thickness of the exponential attenuation in the classical Ekman problem (see, for example, [19]). 
That is, approximately in two inertial periods and, interestingly, regardless of the vertical viscosity coefficient value, inertial oscillations are rearranged from being forced by the wind to the free mode. Thus, the forced solution with exponential attenuation in depth over time $2 / f$ is reconstructed to a form close to linear and the transmission of viscous perturbations in depth decreases many times. In this case, the solution of problem (5) and (7) with $t \geq t_{15}+t_{E}$ approximately can be written for the components of the velocity as

$$
\begin{aligned}
& u_{2}(z, t) \approx\left|W_{2}(z, t)\right| \cos (f t), \\
& v_{2}(z, t) \approx\left|W_{2}(z, t)\right| \sin (f t) .
\end{aligned}
$$

From (13), it follows that when wind action ceases, the inertial components of the velocity of currents are directly proportional to sine and cosine, and therefore the phase shift between the components of velocity is $\pi / 2$. At the same time, free inertial oscillations, according to their form (13), do not appear in the velocity module. This confirms the well-known position that they are not manifested in the power industry, since the Coriolis force is perpendicular to the velocity vector and does not carry out work [19].

Fig. 5 shows the solution of equations (8), (9), (12) and (13) for friction stress, corresponding to wind speed $W_{10}=15 \mathrm{~m} / \mathrm{s}$ and vertical diffusion coefficient $\mathrm{v}=20 \mathrm{~cm}^{2} / \mathrm{s}$, as suitable for strong storm conditions at calculating it using the INMOM model. It can be seen that the inertial oscillations under the action of a constant wind attenuate, tending to a stationary solution of the Ekman problem [19], when at the sea surface the velocity of currents is directed at an angle of $45^{\circ}$ to the wind. In this case, the velocity module of inertial currents in the presence of wind also oscillates with the inertial frequency in exact accordance with the solution (8). At that, if at the surface these module oscillations are close to the harmonic ones, then with depth their harmony is disturbed. This is fully consistent with the calculations for the INMOM model (Fig. 3d). After the termination of wind exposure, the velocity module does not oscillate in exact accordance with solution (12), (13). At the same time, the amplitude (read - the kinetic energy) of inertial movements in the upper layers decreases and increases in the deep layers, i.e., the oscillation energy (barotropization) is redistributed due to vertical viscosity processes when the energy of the average Ekman motion is redistributed from the upper layers to the lower ones. At the same time, there is a curious fact: from a certain characteristic depth (in this case, $10 \mathrm{~m}$ ) in the lower layers, the energy of inertial motions becomes greater than before the cessation of wind exposure.

Solutions for speeds (8) and (11), the graphs of which are shown in Fig. 5, have two time scales. The first, short scale (about two inertial periods) is determined by the vertical viscosity with the coefficient $v$ and is responsible for the formation of a new quasistationary state. At the same time, as was shown above, this scale does not depend on the viscosity itself. Another longer scale of attenuation of inertial oscillations is associated with the drain of energy of quasistationary movements, more intense in the upper layers, into the deep layers of the sea. According to the form of analytical solutions (9), (10) and (12), this attenuation at large time is approximately proportional to the square root of time. 


\section{Conclusions}

The paper presents an analysis of the results of numerical simulation of the Black Sea in the period of a strong storm, exciting inertial oscillations. Their physical interpretation is given and the analytical solution of the system of equations describing them is studied.

The analysis of inertial oscillations based on the results of numerical simulation using the INMOM hydrodynamic model and the WRF atmospheric model showed that these oscillations make a significant contribution to the formation of the currents velocity field and their excitation is caused by a sharp change in the value of the wind speed module. At the same time, they have practically no effect on the change in the depth of the jump in density and sea level.

Analysis of the vertical structure of inertial oscillations showed that there is a separation of inertial motions in amplitude and phase at different depths in the surface layer. With the depth, the amplitudes of the inertial oscillations are grouped in quasi-homogeneous layers, and the harmonic nature of the amplitudes is distorted.

By means of analytical solutions, it is shown that inertial oscillations in the velocity module appear only under wind pump. At the same time, their harmonic character is disturbed with depth. After the wind termination, the velocity of the currents is barotropized by a rapid restructuring from one quasistationary state to another for approximately two inertial periods. In this case, the amplitude of inertial motions in the upper layers decreases, and in the deep ones it increases so that, with a certain characteristic depth in the lower layers, the energy of inertial motions becomes even greater than before the wind stops.

The fact that the inertial frequency is manifested in the module of the water movement velocity with a compelling force of the wind, has a simple physical explanation. Since proper inertial movements occur in the Lagrangian interpretation along a circle, in the case when the speeds of these movements coincide with the direction of the wind, their increase occurs, while the opposite - a decrease.

It is shown that inertial movements have two time scales determined by vertical viscosity. The first - the short scale, is responsible for the formation of a new quasistationary state. It does not depend on the vertical viscosity coefficient and is approximately equal to two inertial periods. Another one - the longer scale is associated with the energy drain of quasistationary inertial oscillations from the upper to the deep layers of the sea. This slow attenuation process is proportional to the square root of time.

\section{REFERENCES}

1. Diansky, N.A. and Fomin, V.V., 2016. Features of Inertial Currents during the Storm on March 23-28, 2013 in the North-Eastern Part of the Black Sea. Processes in Geomedia, 1(5). pp. 37-47. Available at: http://www.geomediacenter.ru/media/PGM_1(5)_2016.pdf [Accessed: 15 October 2018] (in Russian).

2. Ivanov, V.A. and Belocopytov, V.N., 2011. Black Sea Oceanography. Sevastopol: MHI, 209 p.

3. Zatsepin, A.G., Kremenetskiy, V.V., Stanichnyi, S.V. and Burdyugov, V.M., 2010. Black Sea Basin-Scale Circulation and Mesoscale Dynamics under Wind Forcing. In: A.V. Frolov and Yu.D. Resnyansky, eds., 2010. Modern Problems of Ocean and Atmosphere Dynamics: The Pavel S. Lineykin Memorial Volume. Moscow: Triada LTD, pp. 347-368 (in Russian). 
4. Copernicus Marine Environment Monitoring Service. 2019. [online] Available at: http://www.myocean.eu [Accessed: 15 October 2018].

5. Zatsepin, A.G., Ginzburg, A.I., Kostyanoy, A.G., Kremenetskiy, V.V., Krivosheya, V.G., Poyarkov, S.G., Ratner, Yu.B., Skirta, A.Yu., Soloviev, D.M., Stanichny, S.V., Stroganov, O.Yu., Sheremet, N.A. and Yakubenko, V.G., 2002. Variability of Water Dynamics in the Northeastern Black Sea and Its Effect on the Water Exchange between the Near-Shore Zone and Open Basin. Oceanology, 42(suppl. 1), pp. 1-15.

6. Zhurbas, V.M., Zatsepin, A.G., Grigor'eva, Yu.V., Eremeev, V.N., Kremenetsky, V.V., Motyzhev, S.V., Poyarkov, S.G., Poulain, P.-M., Stanichny, S.V. and Soloviev, D.M., 2004. Water Circulation and Characteristics of Currents of Different Scales in the Upper Layer of the Black Sea from Drifter Data. Oceanology, 44(1), pp. 30-44.

7. Diansky, N.A., 2012. Modelirovanie Cirkulyacii Okeana i Issledovanie Ego Reakcii na Korotko-Periodnye $i$ Dolgoperiodnye Atmosfernye Vozdejstviya [Simulation of Ocean Circulation and the Study of Its Response to Short-Term and Long-Term Atmospheric Effects]. Moscow: Fizmatlit, 272 p. (in Russian).

8. Skamarock, W.C., Klemp, J.B., Dudhia, J., Gill, D.O., Barker, D.M., Duda, M.G., Huang, X.Y., Wang, W. and Powers, J.G., 2008. A Description of the Advanced Research WRF Version 3. NCAR Technical Note. Boulder, USA: National Center for Atmospheric Research, $125 \mathrm{p}$. doi:10.5065/D68S4MVH

9. Zalesny, B., Diansky, N.A., Fomin, V.V., Moshonkin, S.N. and Demyshev, S.G., 2012. Numerical Model of the Circulation of the Black Sea and the Sea of Azov. Russian Journal of Numerical Analysis and Mathematical Modelling, [e-journal] 27(1), pp. 95-112. doi:10.1515/rnam-2012-0006

10. Mamaev, O.I., 1986. Morskie Techeniya [Sea Currents]. Moscow: MSU publishing, 104 p. (in Russian).

11. Korotaev, G.K. and Sabinin, K.D., 2017. Inertial Oscillations on the Sheared Current of an Arbitrary Profile. Doklady Earth Sciences, [e-journal] 475(1), pp. 816-817. https://doi.org/10.1134/S1028334X17070145

12. Sabinin, K.D. and Korotaev, G.K., 2017. Inertial Oscillations Over the Background of Shear Currents in the Ocean. Izvestiya, Atmospheric and Oceanic Physics, [e-journal] 53(3), pp. 352-358. doi:10.1134/S0001433817030100

13. Korotaev, G.K., 2018. Inertial Oscillations and the Galilean Transformation. Izvestiya, Atmospheric and Oceanic Physics, [e-journal] 54(2), pp. 201-205. https://doi.org/10.1134/S0001433818020147

14. Bondur, V.G., Sabinin, K.D. and Grebenyuk, Yu.V., 2017. Characteristics of Inertial Oscillations According to the Experimental Measurements of Currents on the Russian Shelf of the Black Sea. Izvestiya, Atmospheric and Oceanic Physics, [e-journal] 53(1), pp. 120-126. https://doi.org/10.1134/S0001433816050030

15. Ball, F.K., 1963. Some General Theorems Concerning the Finite Motions of a Shallow Rotating Liquid Lying on a Paraboloid. Journal of Fluid Mechanics, [e-journal] 17(2), pp. 240-256. https://doi.org/10.1017/S0022112063001270

16. Pollard, R.T. and Millard Jr., R.C., 1970. Comparison between Observed and Simulated Wind-Generated Inertial Oscillations. Deep-Sea Research and Oceanographic Abstracts, [ejournal] 17(4), pp. 813-816. https://doi.org/10.1016/0011-7471(70)90043-4

17. Halpern, D., 1980. Variability of Near-Surface Currents in the Atlantic North Equatorial Countercurrent during GATE. Journal of Physical Oceanography, [e-journal] 10(8), pp. 1213-1220. https://doi.org/10.1175/1520-0485(1980)010<1213:VONSCI >2.0.CO;2

18. Tikhonov, A.N. and Samarskii, A.A., 1990. Equations of Mathematical Physics. New York: Dover Publications, 785 p.

19. Shuleikin, V.V., 1968. Fizika Morya [Physics of the Sea]. Moscow: Nauka, 1090 p. (in Russian). 
About authors:

Nikolay A. Diansky - Chief Research Associate, Lomonosov Moscow State University (build.2, GSP-1, Leninskie Gory, Moscow, 119991, Russian Federation), Dr.Sci. (Phys.-Math.), Senior Lecturer, ORCID ID: 0000- 0002-6785-1956, ResearcherID: R-8307-2018, nikolay.diansky@gmail.com

Vladimir V. Fomin - Senior Research Associate, N.N. Zubov State Oceanographic Institute (6, Kropotkinskiy Ave., Moscow, 119034, Russian Federation), ORCID ID: 0000-0001- 8857-1518, ResearcherID: C-2124-2017, vladimirfomin@ gmail.com

Alexander V. Grigoriev - Leading Research Associate, N.N. Zubov State Oceanographic Institute (6, Kropotkinskiy Ave., Moscow, 119034, Russian Federation), Ph.D. (Phys.-Math.), ORCID ID: 0000-0001-9630-3986, ag-privat@mail.ru

Andrey V. Chaplygin - 6th year student of the Department of Computational Technologies and Modeling, Lomonosov Moscow State University (build.2, GSP-1, Leninskie Gory, Moscow, 119991, Russian Federation), achaplygin99@gmail.com

Andrey G. Zatsepin - Chief Research Associate, Head of the Ocean Dynamics Department, Shirshov Institute of Oceanology, Russian Academy of Science (36 Nahimovskiy pr., Moscow, 117997, Russian Federation), Dr.Sci. (Phys.-Math.), ORCID ID: 0000-0002-5527-5234, Scopus Author ID: 7004260979, ResearcherID: E-4999-2014, zatsepin@ocean.ru

Contribution of the co-authors:

Nikolay A. Diansky - general scientific research supervision; initiation of the research; formulation of the aims and objectives of the study; development and scientific substantiation of the concept; the concept study; selection and justification of numerical methods for solving equations and discussion of the work results

Vladimir V. Fomin - carrying out the numerical calculations using the Black Sea circulation model; preparation of graphic material for the visualization of numerical calculations; analysis of the storm situation simulation; discussion of the work results

Alexander V. Grigoriev - the concept development; analysis and synthesis of the research results; participation in the discussion of the article; the literature review; solution of organizational and technical issues for the text preparation

Andrey V. Chaplygin - analytical solution of the problem of inertial oscillations; development of a mathematical model; drawing analytical solutions for a given wind and its absence; discussion of the work results

Andrey G. Zatsepin - advisory assistance, participation in discussion of the article; discussion of the work results; editing and supplementation of the article text

All the authors have read and approved the final manuscript.

The authors declare that they have no conflict of interest. 